\title{
Bias-Free, Low Power and Optically Driven InP on SOI Switch for Remotely Configurable Photonic Packet Switches
}

\author{
O. $\operatorname{Raz}^{1}$, M. Tassaert ${ }^{2}$, G. Roelkens ${ }^{2}$, D. van Thourhout ${ }^{2}$, H.J.S. Dorren ${ }^{1}$ \\ 1. Eindhoven University of Technology, Den Dolech 2, 5600MB, Eindhoven, The Netherlands \\ 2. INTEC, Ghent University_IMEC, St-Pietersnieuwstraat 41, 9000 Ghent, Belgium
}

\begin{abstract}
First ever demonstration of an InP-on-SOI switch for all-optical-packet-switching. The switch has 300/1300psec on/off switching times, $>30 \mathrm{~dB}$ extinction-ratio and no measurable pattern dependence or switch related power penalties up to a bit rate of $40 \mathrm{~Gb} / \mathrm{sec}$.
\end{abstract}

OCIS codes: (060.1155) All-optical networks; (060.6719) Switching, packet; (130.3120) Integrated optics devices

\section{Introduction}

The exponential growth in packetized data communication has resulted in enormous systems being built for the sake of packet routing. These systems consume large amounts of energy and floor space where they are installed [1]. This growth trend has triggered a large effort over the years into building all optical packet switches which would replace the electronic ones. Both pure all optical switches and hybrid electro-optic solutions have been investigated over the years [2]. On the hybrid front, various broadcast and select switch architectures have been investigated with large arrays of electrically driven Mach-Zehnder Interferometer (MZI) switches [3] or Semiconductor Optical Amplifiers (SOAs) [4]. Also pure all-optical solutions have been extensively researched. In these demonstrations an optical signal was used to switch another device by altering its resonance condition, phase or other critical property. These demonstrations relied on either compact electrically powered integrated devices such as MZI-SOAs [5], or on unbiased cumbersome fiber based switches which exploited fiber non-linearities and required therefore very high optical powers for the control signals [6].

In this paper we report for the $1^{\text {st }}$ time on the use of a membrane InP switch integrated on top of an Silicon on Insulator (SOI) circuit as an optically driven, bias-free, low power and compact packet switch. Low power switching signals $(<2 \mathrm{mWatts})$ were used to drive the switch between high absorption and transparency with more than 30dB extinction ratio and rise and fall times measuring 300 and 1300 psec respectively. Switching of 20 and 40Gb/sec NRZ-OOK data packets was accomplished with the switch showing no pattern dependence or any power penalty related to the switch operation.

\section{Device description and characteristics}

The InP membrane switch is made by bonding a thin $(<100 \mathrm{~nm})$ layer of Epitaxially grown stack of Quantum Wells and further post processing of the membrane to leave a $2 \mu \mathrm{m}$ wide and $150 \mu \mathrm{m}$ long InP stripe on top of an $\mathrm{SiO} 2$ dielectric. The optical signals are vertically injected into the SOI waveguide through near-vertical grating couplers and then transferred from the SOI waveguide to the InP switch structure by using a vertically positioned inverted taper with appropriate lengths in both the SOI and InP layers. In Figure 1 a picture of the fabricated device is shown.
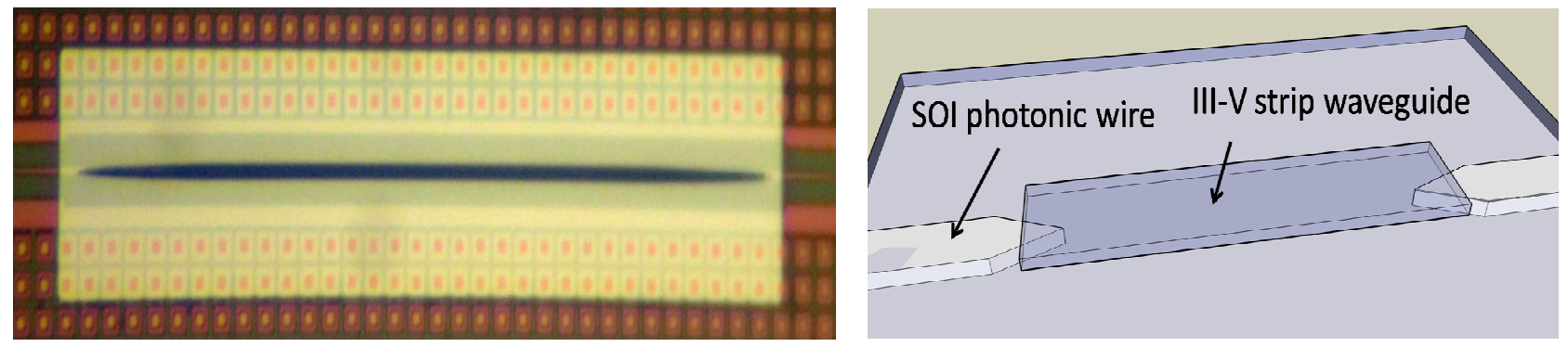

Figure 1: (Left)Top view of fabricated device with length of $150 \mu \mathrm{m}$;(Right) Schematic layout of InP on SOI switch

The InGaAs QWs are designed with a band gap of $1.58 \mu \mathrm{m}$. This means that light absorption is critically dependent on the wavelength, and since the membrane has such a small volume (QW layers: $7.2 \mu \mathrm{m} 3$, total structure: $30 \mu \mathrm{m} 3$ ) very low optical powers can render the InP structure transparent (see Figure 2). 


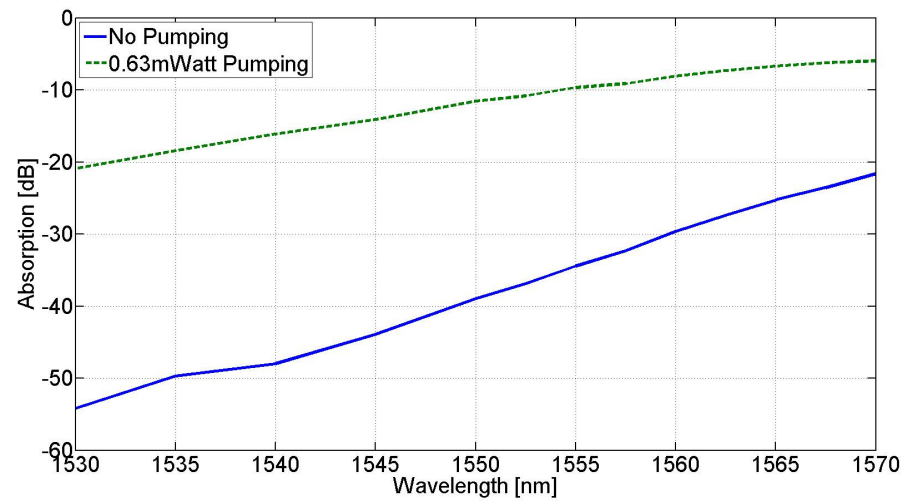

Figure 2: InP switch losses Vs wavelength with and w/o pumping

This effect can be further exploited by using one light source with energy well above the band gap $(1505 \mathrm{~nm})$ to bleach the absorption of another wavelength which is placed closer to the band edge (1542nm). In this way high extinction ratio $(>30 \mathrm{~dB}$, see Figure 3 (right)) and low insertion losses under pumping can be obtained with switching times which vary between $300 \mathrm{psec}$ for on switching and $1.3 \mathrm{nsec}$ for off switching (Figure 3 (left)).
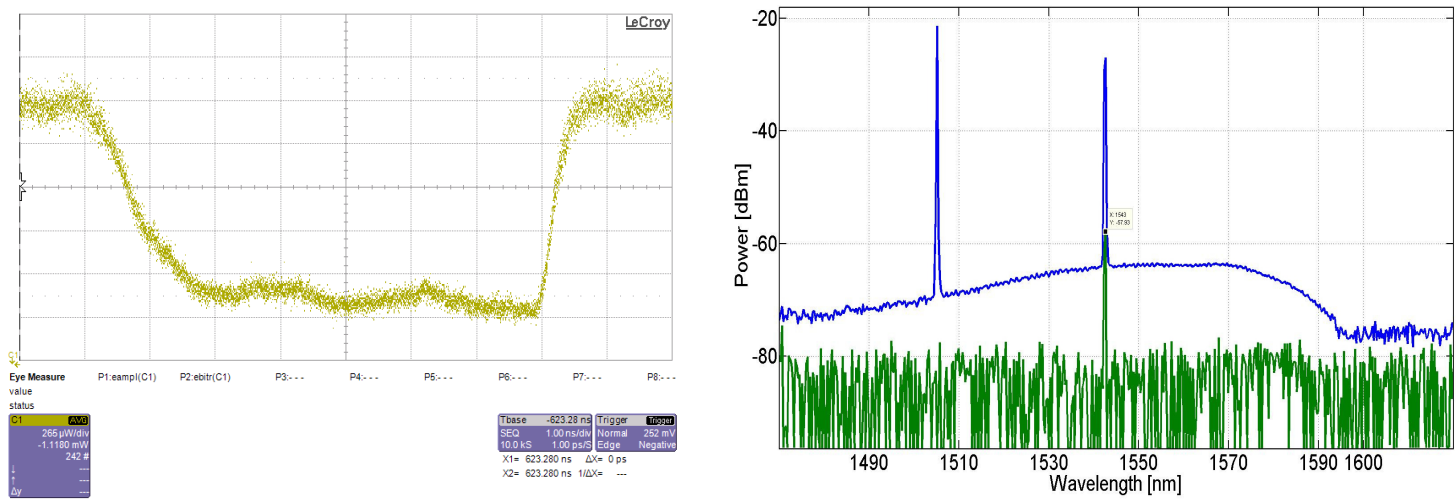

Figure 3: (Left) Switching window as measured for the 1542nm signal; (Right) optical spectra of the signals at the output of the switch showing the extinction ratio, as well as the couplers bandwidth

\section{Switch operation as packet switch}

The switch exhibits high extinction ratio $(>30 \mathrm{~dB}$ ) and an insertion loss of $\sim 19 \mathrm{~dB}$, when optically pumped, with the majority of it coming from the $7.5 \mathrm{~dB}$ loss per grating coupler of the structure. In order to demonstrate the operation of the InP membrane device for packet switching we have used the set-up depicted in Figure 4.

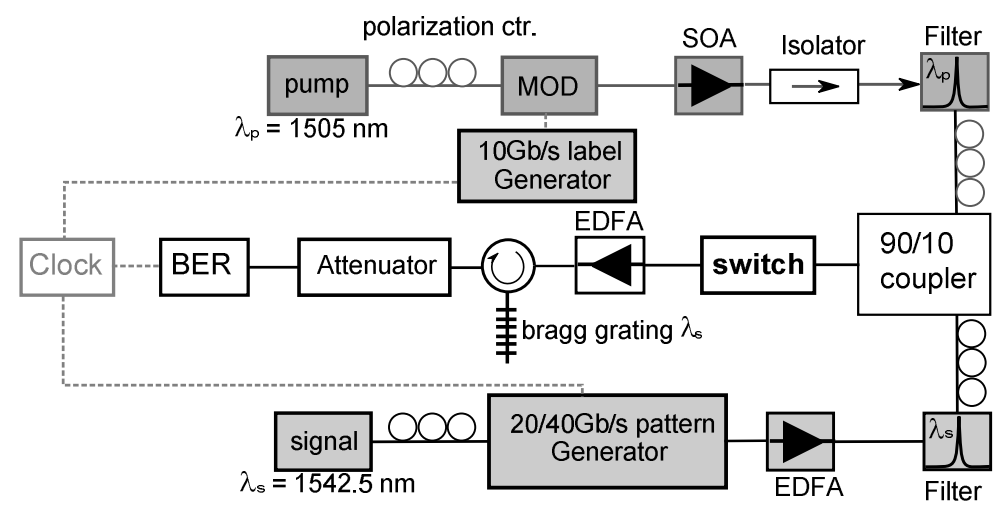

Figure 4: Experimental Set-up

Pump and data wavelengths are used for the demonstration. The data signal with a power of $-12 \mathrm{dBm}$ (in the SOI waveguide) is modulated with a 20/40 Gb/sec Pulse Pattern Generator (PPG) with changing PRBS sequences (for 
the case of the switch being constantly on) and a high utilization $195 / 5$ nsec packets with payload consisting of $2^{15}-1$ PRBS. The pump signal is modulated with a $10 \mathrm{~Gb} / \mathrm{sec}$ modulator and a $10 \mathrm{~Gb} / \mathrm{sec}$ pattern generator (to insure fast electrical and electro-optical response) programmed to 195/5 on/off pulses to toggle the switch state. The pump signal is further amplified to give $+2 \mathrm{dBm}$ (in the SOI waveguide) using an SOA and filtered using a FP filter to avoid accidental pumping of the switch by ASE from the SOA, when the pump signal is off. The performance of the switch was characterized 1st for different length PRBS sequences under constant pumping, to check for any pattern dependence. From Figure 5 (Left), it is clear that no such dependence exists. Furthermore, the signal traveling through the switch shows no receiver sensitivity penalty.
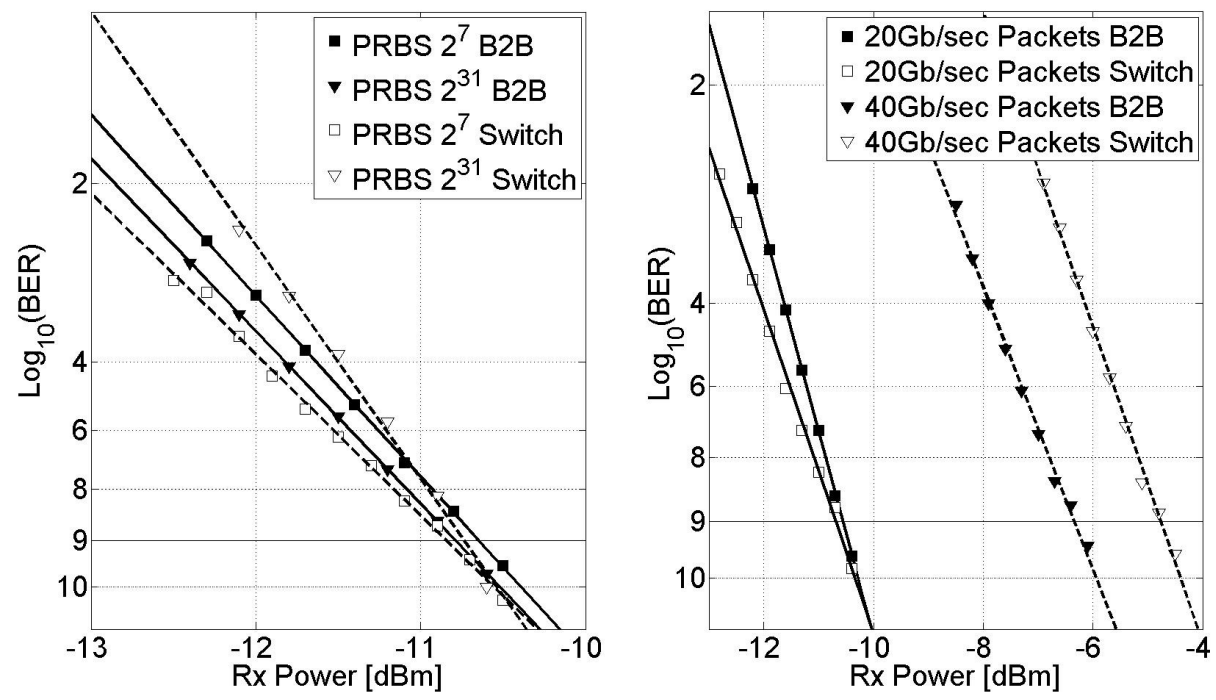

Figure 5: Measured BER as a function of power hitting the receiver

Next the pattern generators were reprogrammed to give the $195 / 5 \mathrm{nsec}$ packets pattern at 20 and $40 \mathrm{~Gb} / \mathrm{sec}$. As can be seen from Figure 5 (right), no receiver penalty is observed for the $20 \mathrm{~Gb} / \mathrm{sec}$ packets, and the measured $1.5 \mathrm{~dB}$ penalty for the $40 \mathrm{~Gb} / \mathrm{sec}$ signal is associated with the limited output power $(<-27 \mathrm{dBm})$ at the SOI circuits output which resulted in degraded OSNR after the required EDFA and hence poorer receiver performance.

\section{Discussion and Conclusions}

We demonstrated penalty free all optical switching of 20 and $40 \mathrm{~Gb} / \mathrm{sec}$ packets using a bias-free membrane InP switch. The demonstrated InP membrane switch is shown to be switchable with very low optical pumping levels. Once the high losses of the grating couplers are resolved, a simple directly modulated laser at the appropriate wavelength can be used for generating the pumping signal. The data signal with its very low power level indicates that the switch can also be remotely controlled since attenuation due to signal propagation of up to $10-15 \mathrm{~dB}$ can be tolerated. While this demonstration concentrated on the single switch operation, more elaborate designs (under work) can make use of multiple pumps and wavelength selective structures to make a $1 \mathrm{xN}$ switch with similar performance.

\section{References}

[1] http://www.cisco.com/en/US/docs/routers/crs/hardware_doc/roadmap/17014hdg.html

[2] S.J. Ben Yoo, "Optical Packet and Burst Switching Technologies for the Future Photonic Internet", J. of Lightwave Tech,Vol. 24, No. 12, pp. 4468- 4492 (2006)

[3] N. Calabretta, W. Wang, T. Ditewig, O. Raz, F. Gomez Agis, S. Zhang H. de Waardt, H.J.S. Dorren, "Scalable Optical Packet Switches for Multiple Data Formats and Data Rates Packets", IEEE Phot. Tech. Lett. , vol. 22, no. 7 pp.483-485, (2010)

[4] Aaron Albores-Mejia, Fausto Gomez-Agis, Harm J. S. Dorren, Xaveer J. M. Leijtens, Tjibbe de Vries, Yok-Siang Oei, Martijn J. R. Heck, Richard Nötzel, D. J. Robbins, Meint K. Smit, and Kevin A. Williams, "Monolithic Multistage Optoelectronic Switch Circuit Routing 160 Gb/s Line-Rate Data“,J. of Lightwave Tech, Vol. 28, Issue 20, pp. 2984-2992 (2010)

[5] N. Calabretta, H.D. Jung, J.H. Llorente, E. Tangdiongga, T. Koonen, H. Dorren, ” All-Optical Techniques Enabling Packet Switching with Label Processing and Label Rewriting", J. of Telecommunications and information technology, pp.20-28 (2009)

[6] Jay E. Sharping, Marco Fiorentino, Prem Kumar, and Robert S. Windeler, "All-Optical Switching Based on Cross-Phase Modulation in Microstructure Fiber”, IEEE Phot. Tech. Let., Vol. 14, No. 1, pp. 77-79 (2002) 